\title{
DC-Bias and Power Allocation in Cooperative VLC Networks for Joint Information and Energy Transfer
}

\author{
Mohanad Obeed, Student Member, IEEE, Hayssam Dahrouj, Senior Member, IEEE, Anas M. Salhab, Senior \\ Member, IEEE, Salam A. Zummo, Senior Member, IEEE, and Mohamed-Slim Alouini, Fellow, IEEE
}

\begin{abstract}
Visible light communications (VLC) have emerged as a strong candidate for meeting the escalating demand for high data rates. In this paper, we consider a VLC network, where multiple access points (APs) serve both energy-harvesting users (EHUs), i.e., users who harvest energy from light emitted by diodes and information users (IUs), i.e., users who gather data information. In order to jointly balance the achievable sum rate at the IUs and the energy harvested by the EHUs, the paper considers maximizing a network-wide utility, which consists of a weighted sum of the IUs sum rate and the EHUs harvested energy, subject to individual IU rate constraint, individual EHU harvested-energy constraint, and AP power constraints, so as to jointly determine the direct current (DC) bias value at each AP, and the power of the alternating-current (AC) signals of the users. A difficult non-convex optimization problem is solved using an iterative approach which relies on inner convex approximations, and compensates for the used approximations using proper outer-loop updates. The paper further considers solving the special cases of the problem, i.e., maximizing the sum rate, and maximizing the total harvested-energy, both subject to the same constraints. Numerical results highlight the significant performance improvement of the proposed algorithms, and illustrate the impacts of the network parameters on the performance trade-off between the sum rate and harvested-energy.
\end{abstract}

Index Terms-Visible light communication, energy harvesting, sum rate, DC bias, iterative algorithm.

\section{INTRODUCTION}

\section{A. Overview}

The need for high data rates and unregulated spectrum services has pushed the research community to examine visible light communication (VLC)techniques as a complementary technology to the existing radio-frequency (RF) wireless networks in indoor environments. This is especially the case because of the scarcity of the available radio-frequency (RF) spectrum due to ultra-dense network deployment. VLC technology uses the visible portion of the electromagnetic spectrum that is completely untapped, safe, free, and provides a high potential bandwidth for wireless data transmission, while also rejecting the existing RF interference [2], [3].

Part of this work is presented at the IEEE Global Communications Conference (IEEE Globecom, Abu Dhabi, UAE, Dec. 9-13, 2018) [1].

M. Obeed, A. M. Salhab, and S. A. Zummo are with the Department Electrical Engineering, King Fahd University of Petroleum \& Minerals (KFUPM), Dhahran, Eastern Province, Saudi Arabia (email: g201106250@kfupm.edu.sa, salhab@kfupm.edu.sa, zummo@kfupm.edu.sa).

H. Dahrouj is with the Department of Electrical Engineering, Effat university, Makkah Province, Saudi Arabia (email: hayssam.dahrouj@gmail.com).

M.-S. Alouini is with the Department Computer, Electrical, and Mathematical Siences \& Engineering, King Abdullah University of Science and Technology (KAUST), Thuwal, Makkah Province, Saudi Arabia (email: slim.alouini@kaust.edu.sa)
VLC, further, provides larger energy efficiency, lower battery consumption, and smaller latency as compared to RFbased networks [4], [5]. VLC can be indeed safely used in sensitive environments such as chemical plants, aircraft, and hospitals [6]. In spite of the small coverage of the transmitters in VLC systems, an exhaustive reuse of frequency can be implemented, with a relatively small effect on the performance due to the manageable co-channel interference [7]. Despite the aforementioned advantages, VLC networks can still be subject to several performance degradation factors, such as limited coverage, non-line-of-sight (non-LoS), transmission failure, frequent handover, and inter-cell interference.

VLC can be obtained by equipping the VLC transmitter with light emitting diodes (LEDs) that can modify the emitted light intensity according to the input current signal. In addition, a VLC receiver is typically equipped with a photodetector (PD) device that converts the received light intensity into an electrical signal. Data are transmitted using an intensity modulation (IM) scheme at the transmitter, and received using a direct detection (DD) scheme at the receiver (IM/DD) [8]. As a result, the modulation signal has to be real-valued unipolar non-negative. Direct current (DC) is added to the modulation signal to assure a non-negative transmitted signal. This means that the transmitted signal contains an alternatingcurrent (AC) part that carry the information and a DC part that used to avoid negative transmitted signals. For the ones who are interested in I-V and L-I LED's characteristics, the reader can refer to [9]-[11] that help in understanding the LEDs concept.

Another attractive VLC feature of valuable interest is its energy harvesting capabilities, which are best enabled through equipping the VLC receivers with solar cells, so as to directly convert the constant light intensity into current signals without the need for an external power supply [12], and with up to $40 \%$ conversion efficiency [13]. In practical indoor environments, however, two different types of users can typically co-exist, i.e., information users (IUs) for high-speed data communication (such as mobiles, laptops, or tablets) and energy harvesting users (EHUs) for low-power applications (such as Internet-of-things (IoT) devices, sensors, or relays). While IUs are data-hungry devices with specific data rate constraints, EHUs aim at harvesting visible light energy, which is widely available in indoor lighting with potential applications in smart buildings, health monitoring, and sensor devices. This motivates us in this paper to evaluate the benefit of a particular VLC-based scheme which considers the coexistence of both IUs and EHUs, and addresses the problem of jointly 
optimizing and balancing the achievable sum rate at the IUs and the total harvested energy by EHUs, by means of adjusting the DC bias at the VLC access points (APs) and the AC power of the users messages. The achievable sum rate is defined as the summation of the achievable data rates of IUs in the system, and it is a function of the electrical AC power.

\section{B. Related Work}

One of this paper goals is to optimize the achievable sum rate in a VLC system, a topic which is extensively studied in the literature of VLC systems, either by supplementing the network with additional RF APs [14]-[17], or by applying VLC APs cooperation [18]-[22].

The work considered in this paper is particularly related to the problems of jointly maximizing IUs sum rate and EHUs harvested energy. In fact, maximizing the achievable sum rate is investigated widely in the literature of wireless networks [23], [24], and in VLC systems [25], [26]. Due to some unique properties of VLC technology such as the channel characteristics and the LED dynamic range and illumination constraints, maximizing the sum rate in VLC systems is intrinsically different from the one considered in RF-based systems, as VLC systems often impose additional systems constraints, e.g., handover overhead [27], blockage probability [28], users distribution in the floor area [15], and users' fieldof-view (FoV) alignments (if the FoV is adjustable) [2]. For instance, one of the effective solutions for addressing VLC coverage and blockage issues is considered in [21], which proposes deploying multiple cooperative distributed APs so to simultaneously serve multiple users, a scenario that is partially adopted in this current paper which considers simultaneously serving both IUs and EHUs.

From the perspective of harvesting energy capabilities, VLC users that are equipped with solar panels can harvest energy from the received light intensity [29]-[35]. For example, reference [29] verifies experimentally the light energy harvested at the mobile phone, when equipped with a commercial solar panel in indoor environments. The authors in [29] show that the devices that are directly exposed to the indoor light can be charged up to a satisfactory power level. Specifically, authors in [29] report that the overall energy that the solar panel can generate during the day is between $123.5 \mathrm{mAh}$ and 173.4 $\mathrm{mAh}$, depending on the temperature. Reference [36] studies the concept of indoor optical wireless power transfer to solar cells during the darkness hours. By using 42 laser diodes, an optical power of $7.2 \mathrm{~W}$ can be delivered to a 30-m distant solar panel.

In the context of simultaneous power and information transfer, the authors in [37] report that a data rate of $0.5 \mathrm{~Gb} / \mathrm{s}$ can be achieved with a gallium arsenide (GaAs) photovoltaic $(\mathrm{PV})$ cell as a detector for simultaneous information and power transfer. [30] and [31] study a dual-hop hybrid VLC/RF communication system, as a means to extend to the out-ofcoverage user. In [30], [31], the authors show that visible light can be used in the first hop to transfer both data information and energy to the relay which, in turn, forwards the data to the destination using the resulting harvested energy. In [32], [33], the sum rate utility of a VLC system consisting of one AP and $K$ users is maximized, subject to individual quality-ofservice (QoS) constraints. Reference [32] assumes that user $k$ receives the information in their assigned time slot, and receives the power within the time slots that are assigned to other users. Reference [33], on the other hand, proposes solving the problem of allocating the optical intensity and time slots through adopting a loose upper bound on the individual required harvested energy. The authors in [34] characterize the outage performance of a hybrid VLC-RF system, where visible light is used for downlink communication to transfer energy and data to the users, and then the users use the harvested energy to transmit RF signals for uplink communication.

All the above papers use the AC component for harvesting the energy at users, while the DC component of light is readily available to transmit energy. Toward this direction, reference [35] considers a network that enables simultaneous light-wave information and power transfer (SLIPT) and maximizes the harvested energy under QoS constraints, so as to determine the DC in a portion of time, given that DC bias in the remaining time is fixed for the purpose of transferring energy only. Reference [35], however, is restricted to a single transmitter and a single receiver only. The authors in [35], further, do not impose any energy constraints on users operation. The authors in [38] focus on the design of the SLIPT receiver and introduce the possible applications of SLIPT in different communication systems.

\section{Contributions}

Different from the aforementioned references, this paper considers a VLC network, where multiple APs cooperate to serve both EHUs (e.g. sensors or IoT devices), and IUs (e.g. laptops, mobile phones, etc.), so as to best capture the multidiverse applications schemes expected in next generations of wireless networks. The paper then investigates the problem of balancing the achievable sum rate at the IUs and the total harvested energy at the EHUs, by means of adjusting the DC bias at APs and allocating users' AC powers. For mathematical tractability, the paper adopts the zero-forcing (ZF) precoding approach to cancel the interference, similar to [39], [40]. Optimizing the precoding matrix, together with the DC biases and the power of the AC signals, would be a complicated problem. Hence, we assume that the precoding matrix is designed only to cancel the interference among IUs.

To balance the performance between the IUs and the EHUs, the paper formulates the optimization problem which maximizes a weighted sum of the IUs sum rate and the EHUs total harvested energy, under QoS and illumination constraints. The performance of the system is a function of both the DC bias values allocated at each AP, and the powers assigned to the users' messages. One of the paper contributions is to solve such a difficult non-convex optimization problem using an iterative approach, which uses inner convex approximations of the objective and constraints. It then compensates for the approximations using proper outer-loop updates. The paper also proposes a simpler sub-optimal baseline approach, which 


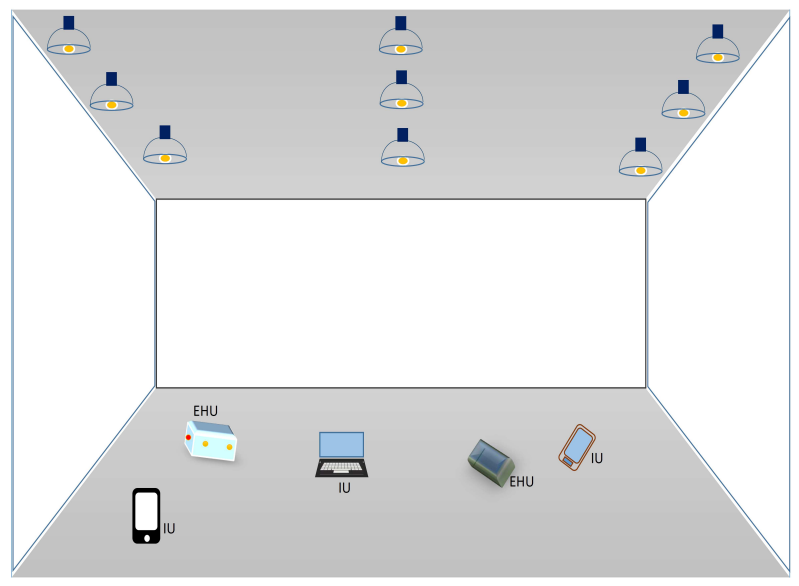

Fig. 1. System model (an example of user distribution when $N_{u, 1}=3$, $N_{u, 2}=2$, and $N_{A}=9$ ).

provides a feasible, yet simple, solution to the formulated problem based on equal DC bias allocation. The paper further considers solving the two special cases of the original optimization, i.e., the problem of maximizing the IUs sum rate, and the problem of maximizing the EHUs total harvested energy, both subject to the same constraints as above. Simulation results highlight the performance and the convergence of our proposed algorithms. They particularly suggest that appreciable harvested energy and sum rate improvement can be reached by optimizing the $\mathrm{DC}$ bias and $\mathrm{AC}$ signal power in VLC systems.

The reminder of this paper is organized as follows. The system model, VLC channel and the energy harvesting channel are presented in Section II. In Section III, we formulate the problem and present the proposed algorithms that solve the formulated problem. We introduce and discuss simulation results in Section IV. The paper is then concluded in Section $\mathrm{V}$.

\section{Notation}

The boldface lowercase letters denote vectors, while boldface uppercase letters denote matrices. Operator $(.)^{T}$ denotes the transpose of the vector or the matrix in parenthesis. Symbol $\mathbb{R}$ denotes the set of real numbers, $\mathbb{R}^{N \times M}$ denotes the set of all $N \times M$ matrices with real elements. Operator $\operatorname{diag}\left(\left[x_{1}, x_{2}, \ldots, x_{N}\right]\right)$ represents a diagonal matrix of diagonal entries $x_{1}, x_{2}, \ldots, x_{N}$. The subscripts $i, j, k$ are used for APs, IUs, and EHUs, respectively. Symbol $\circ$ indicates the vector componentwise multiplication or Hadamard product, while $\oslash$ indicates the vector componentwise division or Hadamard division.

\section{System ANd Channel Models}

\section{A. System Model}

Consider an indoor VLC system consisting of $N_{A}$ VLC APs, which serve $N_{u}$ users in total. Among the $N_{u}$ users, $N_{u, 1}$ users are IUs, and $N_{u, 2}$ are EHUs, i.e., $N_{u}=N_{u, 1}+N_{u, 2}$. Fig. 1 shows an example of the considered system consisting

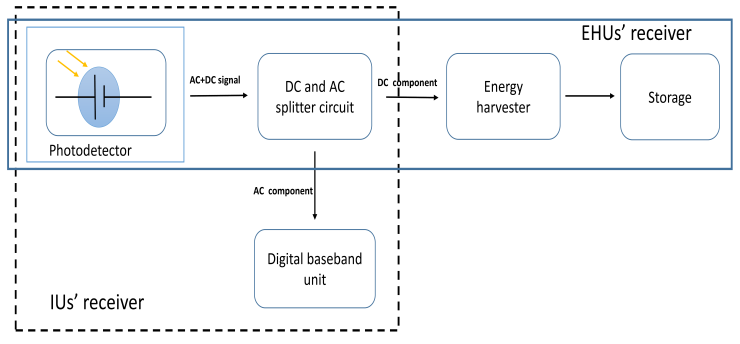

Fig. 2. Receiver Model.

of 9 APs, 2 EHUs, and 3 IUs. We assume that the EHUs are equipped with the functionality of energy harvesting as shown in Fig. 2. Fig. 2 shows that the blocks inside the dashed square represent the receiver of the IUs, while the blocks inside the solid square represent the receiver of EHUs. A capacitor can be used to block the DC componenet and forward the AC component to the data communication circuit, and an inductor can be used to attenuate the AC signal and forward the DC component to the energy harvesting signal. The circuits for AC and DC splitter, energy harvesting, and data communication are studied and presented in [12]. Specifically, Fig. 1, Fig. 2, and Fig. 3 in [12] show the equivalent energy harvesting circuit, the data communication circuit, and the simultaneous energy harvesting and communication, respectively. The paper considers the case where $N_{u, 1}<N_{A}$, and adopts a zeroforcing (ZF) approach to cancel the intra-cell interference, so as to simplify the mathematical tractability of the problem. This assumption, i.e., $N_{u, 1}<N_{A}$, emulates several indoor environments where the number of IUs are less than the number of lamps in the ceiling such as offices, labs, companies ,houses, etc. Let $\mathbf{s} \in \mathbb{R}^{N_{u, 1} \times 1}$ be the vector hosting the information of the $N_{u, 1}$ users, and let $\mathbf{G} \in \mathbb{R}^{N_{A} \times N_{u, 1}}$ be the precoding matrix associated with $\mathbf{s}$. The matrix $\mathbf{G}$ can be written as $\mathbf{G}=\left[\mathbf{g}_{1} \ldots \mathbf{g}_{N_{A}}\right]^{T}$, where $\mathbf{g}_{i} \in \mathbb{R}^{N_{u, 1} \times 1}$ is the $i$ th column of matrix $\mathbf{G}^{T}$. The AC input electrical signal to the AP $i$ can be written as $x_{i}=\mathbf{g}_{i}^{T} \mathbf{\Lambda} \mathbf{s}$, where $\mathbf{s} \in \mathbb{R}^{N_{u, 1} \times 1}$ is the message vector with unit power, $\boldsymbol{\Lambda}=$ $\operatorname{diag}\left(\left[\sqrt{P_{1}}, \sqrt{P_{2}}, \ldots, \sqrt{P_{N_{u, 1}}}\right]\right), P_{j}$ is the AC electrical power allocated to $s_{j}$ (the $j$ th user's message). The DC bias of the $i$ th $\mathrm{AP}$ is denoted by $b_{i}$ and must be added to $x_{i}$ to avoid the resulting non-positive signals [35]. The electrical signal, afterwards, modulates the optical intensity of the lightemitting diodes (LEDs) at AP $i$. The transmitted signal at AP $i$ can, therefore, be written as

$$
y_{t, i}=\rho_{s e}\left(b_{i}+x_{i}\right)
$$

where $\rho_{s e}$ is the slope efficiency of the LED or the electricalto-optical conversion factor of the LED measured in W/A. Let $I_{L}$ and $I_{H}$ be the minimum and the maximum input bias currents, respectively, i.e., $b_{i} \in\left[I_{L}, I_{H}\right]$. To guarantee that the output optical power is a linear function of the input current, the transmitter LED must be in its linear region. To this end, the peak amplitude of the modulated signal $x_{i}$, denoted by $A_{i}$, must satisfy the following constraint

$$
A_{i} \leq \min \left(b_{i}-I_{L}, I_{H}-b_{i}\right) .
$$


Constraint (2) implies that $A_{i}$ must satisfy two constraints, which are $A_{i}+b_{i} \leq I_{H}$ and $I_{L} \leq b_{i}-A_{i}$, to guarantee that the input electrical current to the LED is within the range of the linear region LED operation. It is important to note that restricting the DC bias current to be lower than a given maximum current ensures eye safety. This is because the eye safety constraint can be met by limiting the radiated optical power, which can be controlled by the maximum limit of the input current $I_{H}$.

\section{B. Channel Model}

The paper adopts a LoS VLC channel model as in [41] and the first order reflected path for simplicity. More precisely, the LoS link between the $i$ th LED and the $j$ th user, denoted by $h_{i, j}^{L o S}$, can be written as follows

$$
h_{i, j}^{L o S}=\frac{(m+1) A_{p}}{2 \pi d_{i, j}^{2}} \cos ^{m}\left(\phi_{i, j}\right) g_{o f} f\left(\theta_{i, j}\right) \cos \left(\theta_{i, j}\right),
$$

where $m=-1 / \log _{2}\left[\cos \left(\theta_{\frac{1}{2}}\right]\right.$ is the Lambertian index, $\theta_{\frac{1}{2}}$ is the semi-angle of half power, $A_{p}$ is the photo-detector (PD) physical area, $d_{i, j}$ is the distance between the AP $i$ and the user $j, g_{o f}$ is the optical filter gain, $\phi_{i, j}$ is the radiance angle, $\theta_{i, j}$ is the incidence angle, and $f\left(\theta_{i, j}\right)$ is the optical concentrator gain given by

$$
f\left(\theta_{i, j}\right)= \begin{cases}\frac{n^{2}}{\sin ^{2}(\Theta)}, & \theta_{i, j} \leq \Theta \\ 0, & \theta_{i, j}>\Theta\end{cases}
$$

where $\Theta$ is the the user's FoV semi-angle and $n$ is the refractive index. Reference [42] shows that the DC attenuation of the first reflected link can be expressed as

$$
\begin{array}{r}
d h_{i, j}^{N L o S}=\frac{(m+1) A_{p}}{2 \pi l_{i}^{2} l_{j}^{2}} \varrho \cos ^{m}\left(\phi_{r}\right) \cos \left(\alpha_{1}\right) \cos \left(\alpha_{2}\right) \\
g_{o f} f\left(\theta_{r}\right) \cos \left(\theta_{r}\right) d A_{s},
\end{array}
$$

where $\theta_{r}$ and $\phi_{r}$ are the incidence and irradiance angles of the first reflected link, respectively, $l_{i}^{2}$ and $l_{j}^{2}$ are the distances between the $i$ th transmitter and the reflecting point and between the reflecting point and the $j$ th user, respectively, $d A_{s}$ is the reflective area, $\varrho$ is the reflection factor, respectively, and $\alpha_{1}$ and $\alpha_{2}$ are the the irradiance angles corresponding to the reflecting point and to the $\mathrm{PD}$, respectively. Therefore, the VLC equivalent channel between $i$ th AP and $j$ th user can be expressed as

$$
h_{i, j}=h_{i, j}^{L o S}+h_{i, j}^{N L o S} .
$$

After separating the $\mathrm{DC}$ bias from the $\mathrm{AC}$ signal at the receiver, the signal vector received at the users from all APs ( $\mathbf{Y}_{r}$ with size $\left.N_{u, 1} \times 1\right)$ is given by

$$
\mathbf{Y}_{r}=\rho_{o e} \rho_{s e} \mathbf{H G} \boldsymbol{\Lambda} \mathbf{s}+\mathbf{n},
$$

where $\mathbf{H} \in \mathbb{R}^{N_{u} \times N_{A}}$ is the channel attenuation matrix that is assumed to be known at APs, $\rho_{o e}$ is the optical-to-electric conversion factor, and $\mathbf{n}$ is the noise vector with size $N_{u, 1} \times 1$, which includes the thermal noise and the shot noise at the user, and can be modeled as zero-mean real-valued AWGN with variance $\sigma^{2}=N_{0} W$, where $W$ is the modulation bandwidth, and $N_{0}$ is the noise power spectral density. The precoding matrix $\mathbf{G}$ is used to cancel the inter-cell interference by diagonalizing the channel matrix, i.e., $\mathbf{G}=\mathbf{H}^{T}\left(\mathbf{H} \mathbf{H}^{T}\right)^{-1}$. This means that the received signal at the $j$ th user or the $j$ th element of vector $\mathbf{Y}_{r}$ is given by

$$
y_{r, j}=\rho_{o e} \rho_{s e} \sqrt{P_{j}} s_{j}+n_{j} .
$$

Since the power of $s_{j}$ is normalized, a tight lower bound on the network sum rate at the $N_{u, 1}$ IUs can then be written as [43]

$$
f_{R}(\mathbf{P})=\beta \sum_{j=1}^{N_{u, 1}} \log _{2}\left(1+\frac{e \rho_{o e}^{2} \rho_{s e}^{2} P_{j}}{2 \pi W N_{0}}\right),
$$

where $\mathbf{P}$ is a vector with size $N_{u, 1} \times 1$ hosting the messages powers of the IUs, $\beta=W / 2$ is a constant, and $e$ is the constant exponential (Euler's number). It is important to note that, from (7), we can define the relation between the electrical transmit power at AP $i$ and the assigned powers of the messages as [44]

$$
p_{i}=\sum_{j=1}^{N_{u, 1}} g_{i, j}^{2} P_{j}
$$

where $g_{i, j}$ is the $(i, j)$ th element of matrix $\mathbf{G}$.

\section{Energy Harvesting Signals}

For the EHUs, the DC component of the received signal is passed through an inductor and resistor to the energy harvesting circuit [12], [35]. The harvested energy (per unittime) is given by [29]

$$
E=f I_{s c} V_{o c}
$$

where $f$ is the fill factor (typically around 0.75 ), and $I_{s c}$ is the short-circuit DC current measured by shutting the solar panel charging circuit [29], which is given at the $k$ th energyharvesting user by

$$
I_{s c, k}=\rho_{o e} \rho_{s e} \mathbf{h}_{k}^{T} \mathbf{b}
$$

where $\mathbf{b}=\left[b_{1}, b_{2}, \ldots, b_{N_{A}}\right]^{T}$ is the DC bias vector at APs, $\mathbf{h}_{k}$ is the channel vector from all APs to the user $k$ with size $N_{A} \times 1$, and $V_{o c}$ is the voltage that is measured by opening the solar panel charging circuit and is given by [31], [35]

$$
V_{o c, k}=V_{t} \ln \left(1+\frac{I_{s c, k}}{I_{0}}\right),
$$

where $V_{t}$ is the thermal voltage and $I_{0}$ is the dark saturation current of the PD. Hence, the harvested energy (per unit-time) at user $k$ is given by

$$
E_{k}(\mathbf{b})=f \rho_{o e} \rho_{s e} V_{t} \mathbf{h}_{k}^{T} \mathbf{b} \ln \left(1+\frac{\rho_{o e} \rho_{s e} \mathbf{h}_{k}^{T} \mathbf{b}}{I_{0}}\right),
$$

and the total harvested energy at all $N_{u, 2}$ users is given by

$$
f_{E}(\mathbf{b})=\sum_{k=1}^{N u_{2}} E_{k}(\mathbf{b})
$$




\section{Problem Formulation AND Algorithms}

In order to jointly optimize the achievable sum rate utility at the IUs and the total harvested energy utility at the EHUs, this section motivates and then considers maximizing a weighted sum of both utilities under QoS constraints and maximum transmit power constraint. The section then proposes two different solutions to solve the formulated non-convex problem by efficiently adjusting the DC bias vector and the users' powers. The section finally addresses the two individual optimization problems separately, i.e., maximizing the sum rate utility, and then maximizing the total harvested energy utility, both under the same constraints.

\section{A. Weighted Sum Utility Maximization and Constraints}

The utility function for the IUs is the sum rate that is given in (9), which is a function of the messages' powers, while the utility function for the EHUs, given in (15), is the total harvested energy, which is a function of the APs DC bias.

The first constraint that should be considered is that the DC bias at each AP should be within the maximum and minimum input currents (i.e., $I_{L} \leq b_{i} \leq I_{H} \forall i$ ). It is important to note that the DC bias $b_{i}$ at the $i$ th $\mathrm{AP}$ must be greater than or equal to $\frac{I_{H}+I_{L}}{2}$. This is because decreasing $b_{i}$ to be less than $\frac{I_{H}+I_{L}}{2}$ results in decreasing the harvested energy (14). It also decreases $A_{i}$ (based on (2)), which decreases the transmit power of the signal that leads to a decrease in the sum rate (9). Parameter $b_{i}$, therefore, should satisfy $b_{i} \geq \frac{I_{H}+I_{L}}{2}$, and $\min \left(I_{H}-b_{i}, b_{i}-I_{L}\right)$ becomes equal to $I_{H}-b_{i}$.

The second constraint that should be considered is that the input signal should be positive and within the maximum and minimum allowable limit currents. In other words, the input signal for the $i$ th AP should satisfy the following

$$
I_{L} \leq \mathbf{g}_{i}^{T} \mathbf{\Lambda} \mathbf{s}+b_{i} \leq I_{H}
$$

Constraint (16) can be written equivalently as

$$
\mathbf{g}_{i}^{T} \mathbf{\Lambda} \mathbf{s} \leq I_{H}-b_{i},
$$

and

$$
-\mathbf{g}_{i}^{T} \boldsymbol{\Lambda} \mathbf{s} \leq b_{i}-I_{L} .
$$

Constraints (17) and (18) can be written equivalently as

$$
\left|\mathbf{g}_{i}^{T} \mathbf{\Lambda} \mathbf{s}\right| \leq \min \left(I_{H}-b_{i}, b_{i}-I_{L}\right) .
$$

Constraint $\frac{I_{H}+I_{L}}{2} \leq b_{i} \leq I_{H}$ implies that $\min \left(I_{H}-b_{i}, b_{i}-\right.$ $\left.I_{L}\right)=I_{H}-b_{i}$. Therefore, constraint (19) can be written equivalently as

$$
\left|\mathbf{g}_{i}^{T} \mathbf{\Lambda} \mathbf{s}\right| \leq\left(I_{H}-b_{i}\right) .
$$

If the optimal $b_{i}$ satisfies $b_{i} \leq I_{H}-\left|\mathbf{g}_{i}^{T} \boldsymbol{\Lambda} \mathbf{s}\right|, b_{i}$ can be increased to $b_{i}=I_{H}-\left|\mathbf{g}_{i}^{T} \mathbf{\Lambda} \mathbf{s}\right|$, which increases the objective function without violating the constraints. Hence, the inequality in (20) should be satisfied with equality. In order to represent constraint (20) in terms of power, we should find the expectation of the square of both sides [45]. Because $E\left(\mathbf{s s}^{T}\right)=\mathbf{I}_{N_{u, 1}}$, where $\mathbf{I}_{N_{u, 1}}$ is the identity matrix with size $N_{u, 1} \times N_{u, 1}$, constraint (20) can be represented equivalently as

$$
\mathbf{g}_{i}^{T} \boldsymbol{\Lambda} \boldsymbol{\Lambda}^{T} \mathbf{g}_{i}=\left(I_{H}-b_{i}\right)^{2} .
$$

Since $\Lambda=\operatorname{diag}\left[\sqrt{P_{1}}, \ldots, \sqrt{P_{N_{u, 1}}}\right]$, constraint (21) can be written as

$$
\sum_{j=1}^{N_{u, 1}} g_{i, j}^{2} P_{j}=\left(I_{H}-b_{i}\right)^{2}, \forall i,
$$

where $g_{i, j}$ is the $j$ th component of $\mathbf{g}_{i}$ vector.

Expression (22) shows that the relation between both vectors is not one-to-one. More specifically, a unique DC bias vector $\mathbf{b}$ can be calculated for a given messages' power vector. The messages' power vector $\mathbf{P}$ might, however, have several solutions from a given DC bias vector. Expression (22) also shows that increasing the DC biases increases the total harvested energy at the EHUs, but decreases the data rate at the IUs. Such conflicting impact of the DC bias motivates the need for jointly optimizing both utilities by means of maximizing a weighted-sum under QoS and LEDs' linear operational region constraints. In this formulated problem, the weights of the utility functions can be controlled by a variable, called $\alpha \in[0,1]$. In other words, $\alpha$ is the weight which balances between the sum rate and harvested energy utilities. Mathematically, the considered optimization problem can be formulated as follows

$$
\begin{array}{ll}
\max _{\mathbf{b}, \mathbf{P}} & \alpha f_{R}(\mathbf{P})+\frac{(1-\alpha)}{\omega} f_{E}(\mathbf{b}) \\
\text { s.t. } & \beta \log _{2}\left(1+\frac{e \rho_{o e}^{2} \rho_{s e}^{2} P_{j}}{2 \pi W N_{0}}\right) \geq R_{t h, j}, \\
& j=1, \ldots, N_{u, 1} \\
& f \rho_{o e} \rho_{s e} V_{t} \mathbf{h}_{k}^{T} \mathbf{b} \ln \left(1+\frac{\rho_{o e} \rho_{s e} \mathbf{h}_{k}^{T} \mathbf{b}}{I_{0}}\right) \geq E_{t h, k}, \\
& k=1, \ldots, N_{u, 2} \\
& \sum_{j=1}^{N_{u, 1}} g_{i, j}^{2} P_{j}=\left(I_{H}-b_{i}\right)^{2}, \quad i=1, \ldots, N_{A} \\
& \frac{I_{H}+I_{L}}{2} \leq b_{i} \leq I_{H}, \quad i=1, \ldots, N_{A},
\end{array}
$$

where $R_{t h, j}$ and $E_{t h, k}$ are the minimum required data rate at the $j$ th user and the minimum required energy to be harvested by the $k$ th user, respectively, and $\omega$ is a constant chosen to numerically equalize the order of magnitudes of the functions $f_{R}(\mathbf{P})$ and $\frac{1}{\omega} f_{E}(\mathbf{b})$. Constraints (23b) and (23c) are imposed to satisfy the minimum required fairness among IUs and the EHUs, while constraints in (23d) and (23e) are imposed to avoid any clipping and guarantee that the LEDs operate in their linear region. It is important to note that problem (23) solves three types of problems: 1) maximizing the sum rate, which is achieved when we set $\alpha=1,2$ ) maximizing the total harvested energy, which can be achieved by setting $\alpha=0,3$ ) and maximizing a weighted sum of both utility functions for any $\alpha \in(0,1)$.

Problem (23) cannot be easily solved, since the objective function and the constraint (23c) are not concave, resulting in a difficult non-convex optimization problem. Specifically, $f_{R}(\mathbf{P})$ is a concave function in terms of $\mathbf{P}$, while $f_{E}(\mathbf{b})$ is a convex function in terms of $\mathbf{b}$, which makes their weighted sum a non-concave objective function. This paper next solves problem (23) by first reformulating the problem in a more 
compact form, and then by proposing an numerical iterative approach.

The main idea of the proposed approach is that the problem is first formulated in terms of the messages' power vector $\mathbf{P}$ only, using the relation given in (22). The paper then proposes a heuristic, yet efficient, algorithm to solve the reformulated problem through considering an approximated convex version of the problem, and then by correcting for the approximation in an outer loop update. For the sake of comparison, the paper further proposes a simple baseline approach, which guarantees a feasible solution to (23).

\section{B. Problem Reformulation}

As discussed earlier, a unique DC bias vector $\mathbf{b}$ can be calculated for a given messages' power vector. Thus, to reformulate problem (23) in a more compact fashion, we choose to formulate the objective function and constraints of problem (23) in terms of the vector $\mathbf{P}$ only. Using the relation in (22), the DC bias vector can be expressed as

$$
\mathbf{b}=I_{H} \mathbf{1}_{N_{A}}-\sqrt{\overline{\mathbf{G}} \mathbf{P}},
$$

where $\mathbf{1}_{N_{A}}$ is the unity vector of length $N_{A}$ with all entries set to 1 , the matrix $\overline{\mathbf{G}}$ is defined as $\mathbf{G}=\left[\overline{\mathbf{g}}_{1}, \overline{\mathbf{g}}_{2}, \ldots, \overline{\mathbf{g}}_{N_{A}}\right]^{T}$, with $\overline{\mathbf{g}}_{i}=\left[g_{i, 1}^{2}, g_{i, 2}^{2}, \ldots, g_{i, N_{u, 1}}^{2}\right]^{T}$, and where the square root denotes the componentwise square root of the vector argument.

Plugging (24) in the energy harvesting functions (14) and (15), we obtain

$$
\begin{aligned}
E_{k}(\mathbf{P})=f \rho_{o e} \rho_{s e} & V_{t} \mathbf{h}_{k}^{T}\left(I_{H} \mathbf{1}_{N_{A}}-\sqrt{\overline{\mathbf{G}} \mathbf{P}}\right) \ln (1 \\
+ & \left.\frac{\rho_{o e} \rho_{s e} \mathbf{h}_{k}^{T}\left(I_{H} \mathbf{1}_{N_{A}}-\sqrt{\overline{\mathbf{G}} \mathbf{P}}\right)}{I_{0}}\right),
\end{aligned}
$$

and

$$
f_{E}(\mathbf{P})=\sum_{k=1}^{N_{u, 2}} E_{k}(\mathbf{P}) .
$$

Using (24), the constraints in (23e) can be rewritten as

$$
0 \leq \overline{\mathbf{g}}_{i}^{T} \mathbf{P} \leq\left(\frac{I_{H}-I_{L}}{2}\right)^{2}, \quad i=1, \ldots, N_{A} .
$$

Substituting (25), (26), and (27) in the optimization problem (23), the problem can then be formulated in terms of the messages' power vector as follows

$$
\begin{array}{cl}
\max _{\mathbf{P}} & \alpha f_{R}(\mathbf{P})+\frac{(1-\alpha)}{\omega} f_{E}(\mathbf{P}) \\
\text { s.t. } & P_{j} \geq P_{j, \text { min }}, \quad j=1, \ldots, N_{u, 1} \\
& E_{k}(\mathbf{P}) \geq E_{t h, k}, \quad k=1, \ldots, N_{u, 2} \\
& \overline{\mathbf{g}}_{i}^{T} \mathbf{P} \geq 0, \quad i=1, \ldots, N_{A} \\
& \overline{\mathbf{g}}_{i}^{T} \mathbf{P} \leq\left(\frac{I_{H}-I_{L}}{2}\right)^{2}, i=1, \ldots, N_{A},
\end{array}
$$

where $P_{j, \text { min }}=\frac{\left(2^{\frac{R_{t h, j}}{\beta}}-1\right) 2 \pi W N_{0}}{e \rho_{s e}^{2} \rho_{o e}^{2}}$. Because functions $f_{E}(\mathbf{P})$ and $E_{k}(\mathbf{P})$ are not concave, the problem in (28) is still a non-convex optimization problem. Hence, we next propose a novel method that solves problem (28) by using a proper convex approximation, and then by compensating for the approximation in the outer loop.

\section{Problem Convexification}

To convexify problem (28), we utilize a two-step iterative approach. At the first step, we fix the DC bias vector values for specific terms of the non-concave functions, so as to get rid of the square root and the logarithm function expression in the energy functions. After solving the problem, the second step substitutes the updated value of the DC bias vector in the terms of the non-concave functions. More specifically, in the first step (and at the very first iteration), let $\hat{\mathbf{b}}=\frac{I_{H}+I_{L}}{2} \mathbf{1}_{N_{A}}$ (i.e., $\hat{b}_{i}=\frac{I_{H}+I_{L}}{2}$ ) be the initial DC bias vector. Therefore, the relation in (22) can be approximated as follows

$$
\overline{\mathbf{G}} \mathbf{P} \cong\left(I_{H} \mathbf{1}_{N_{A}}-\mathbf{b}\right) \circ\left(I_{H} \mathbf{1}_{N_{A}}-\hat{\mathbf{b}}\right) .
$$

The DC bias vector can be approximated as follows

$$
\mathbf{b} \cong I_{H} \mathbf{1}_{N_{A}}-\overline{\mathbf{G}} \mathbf{P} \oslash\left(I_{H} \mathbf{1}_{N_{A}}-\hat{\mathbf{b}}\right) .
$$

Define

$$
\mathbf{G}_{b}=\left[\frac{1}{I_{H}-\hat{b}_{1}} \overline{\mathbf{g}}_{1}, \frac{1}{I_{H}-\hat{b}_{2}} \overline{\mathbf{g}}_{2}, \ldots, \frac{1}{I_{H}-\hat{b}_{N_{A}}} \overline{\mathbf{g}}_{N_{A}}\right]^{T},
$$

we can re-write (30) as follows

$$
\mathbf{b} \cong I_{H} \mathbf{1}_{N_{A}}-\mathbf{G}_{b} \mathbf{P} .
$$

To further convexify the energy functions, define $z_{k}(\hat{\mathbf{b}})$ as $z_{k}(\hat{\mathbf{b}})=\ln \left(1+\frac{\rho_{o e} \rho_{s e} \mathbf{h}_{k}^{T} \hat{\mathbf{b}}}{I_{0}}\right)$, which is a constant that depends on $\hat{\mathbf{b}}$.

Problem (28) can now be readily approximated as a convex optimization problem. For the completeness of presentation, define the following variables (which are all functions of the estimated DC bias vector $\hat{\mathbf{b}}$ ):

$\mathbf{x}_{k}=f \rho_{o e} \rho_{s e} V_{t} z_{k}(\hat{\mathbf{b}}) \mathbf{h}_{k}, k=1, \ldots, N_{u, 2}$,

$x=\sum_{k=1}^{N_{u, 2}} \mathbf{x}_{k}^{T}\left(I_{H} \mathbf{1}_{N A}\right)$,

$\mathbf{w}=\sum_{k=1}^{\bar{N}_{u, 2}} \mathbf{x}_{k}^{T} \mathbf{G}_{b}, m_{k}=I_{H} \mathbf{x}_{k} \mathbf{1}_{N_{A}}-E_{t h, k}$, and $\mathbf{w}_{k}=\mathbf{x}_{k}^{T} \mathbf{G}_{b}, k=1, \ldots, N_{u, 2}$.

Using the above notations, problem (28) can be approximated as follows

$$
\begin{array}{cl}
\max _{\mathbf{P}} & \alpha f_{R}(\mathbf{P})+\frac{(1-\alpha)}{\omega}\left(x-\mathbf{w}^{T} \mathbf{P}\right) \\
\text { s.t. } & P_{j} \geq P_{j, m i n}, \quad j=1, \ldots, N_{u, 1} \\
& \mathbf{w}_{k}^{T} \mathbf{P} \leq m_{k}, \quad k=1, \ldots, N_{u, 2} \\
& \overline{\mathbf{g}}_{i}^{T} \mathbf{P} \geq 0, \quad i=1, \ldots, N_{A} \\
& \overline{\mathbf{g}}_{i}^{T} \mathbf{P} \leq\left(\frac{I_{H}-I_{L}}{2}\right)^{2}, i=1, \ldots, N_{A},
\end{array}
$$

Since the function $f_{R}(\mathbf{P})$ is concave and the function $x-\mathbf{w}^{T} \mathbf{P}$ is linear, the objective function in (32) is concave. Furthermore, all the constraints in (32) are linear, which means that the optimization problem (32) is convex and, thus, can be solved using efficient algorithms [46]. We next characterize the optimal solution of problem (32) by deriving the firstorder Karush-Kuhn-Tucker (KKT) conditions, which helps iteratively finding the primal and dual variables associated with problem (32). 
Proposition 1. The solution of problem (32) is given by

$$
\begin{array}{r}
P_{j}=\frac{-\alpha \beta}{\ln (2)\left[-\frac{1}{\omega}(1-\alpha) \mathbf{w}(j)+\lambda_{j}-\sum_{k=1}^{N_{u}, 2} \mu_{k} \mathbf{w}_{k}(j)-\sum_{i=1}^{N_{A}} d_{i} \overline{\mathbf{g}}_{i}(j)\right]} \\
-\frac{1}{\gamma}, j=1, \ldots, N_{u, 1}, \quad \text { (33) }
\end{array}
$$

where $\gamma=\frac{e \rho_{o e}^{2} \rho_{s e}^{2} P}{2 \pi W N_{0}}, \lambda_{j}, \mu_{k}$, and $d_{i}$ are the dual variables associated with constraints (32b), (32c), and (32e), respectively, $p_{\max }=\left(\frac{I_{H}-I_{L}}{2}\right)^{2}$ is the electrical maximum transmit power, and $\mathrm{w}(j)$ denotes to the jth element of the vector $\mathbf{w}$.

Proof. The proof hinges upon the interpretation of the Lagrangian duality of problem (32). Observe first that constraints in (32d) are rather redundant, since all the elements in $\overline{\mathbf{g}}_{i}$ are positive, $\forall i=1, \ldots, N_{A}$, and since the values of the vector $\mathbf{P}$ are guaranteed to be positive by constraints (32b). The Lagrangian function of problem in (32) can, therefore, be expressed as follows

$$
\begin{array}{r}
\zeta=-\alpha \beta \sum_{j=1}^{N_{u, 1}} \log \left(1+\gamma P_{j}\right)-\frac{(1-\alpha)}{\omega}\left(x-\mathbf{w}^{T} \mathbf{P}\right) \\
-\sum_{j=1}^{N_{u, 1}} \lambda_{j}\left(P_{j}-P_{j, \text { min }}\right)+\sum_{k=1}^{N_{u, 2}} \mu_{k}\left(\mathbf{w}_{k}^{T} \mathbf{P}-m_{k}\right) \\
+\sum_{i=1}^{N_{A}} d_{i}\left(\overline{\mathbf{g}}_{i}^{T} \mathbf{P}-p_{\max }\right) .
\end{array}
$$

Based on first-order KKT conditions [46], we have

$$
\frac{\partial \zeta}{\partial P_{j}}=0, j=1, \ldots, N_{u, 1} .
$$

Solving (35), we obtain

$$
\begin{array}{r}
-\alpha \beta \frac{\gamma}{\ln (2)\left(1+\gamma P_{j}\right)}+\frac{1}{\omega}(1-\alpha) \mathbf{w}(j)-\lambda_{j} \\
\quad+\sum_{k=1}^{N_{u, 2}} \mu_{k} \mathbf{w}_{k}(j)+\sum_{i=1}^{N_{A}} d_{i} \overline{\mathbf{g}}_{i}(j)=0 .
\end{array}
$$

Re-ordering (36) then gives (33), which completes the proof.

The dual variables $\lambda_{j}, \mu_{k}$, and $d_{i}$ must be selected in such a way that the resulted allocated power vector achieves the associated constraints. For instance, the value of the dual variables $\lambda_{j}$ must be selected to achieve the $j$ th constraint in (32b). $\lambda_{j}$ can in fact be found after substituting (33) in constraints (32b), which gives the following

$$
\begin{aligned}
\lambda_{j} \leq \frac{-\alpha \beta}{\ln (2)\left(P_{j, m i n}\right.}+ & \left.\frac{1}{\gamma}\right) \\
& +\frac{1}{\omega}(1-\alpha) \mathbf{w}(j) \\
& \left.+\sum_{k=1}^{N_{u, 2}} \mu_{k} \mathbf{w}_{k}(j)+\sum_{i=1}^{N_{A}} d_{i} \overline{\mathbf{g}}_{i}(j)\right) .
\end{aligned}
$$

The other dual variables, i.e., $\mu_{k}$ and $d_{i}$, can be found by using the subgradient method. More specifically, for a fixed value of $P_{j}$ (i.e., using (33) based on preset dual variables values), the subgradient method iteratively updates the values of $\mu_{k}$ and $d_{i}$ as follows

$$
\begin{aligned}
& \mu_{k}(n+1)=\mu_{k}(n)+\delta_{\mu}\left(\mathbf{w}_{k}^{T} \mathbf{P}-m_{k}\right), j=1, \ldots, N_{k}, \\
& d_{i}(n+1)=d_{i}(n)+\delta_{d}\left(\overline{\mathbf{g}}_{i}^{T} \mathbf{P}-p_{\max }\right), i=1, \ldots, N_{A},
\end{aligned}
$$

where $\delta_{\mu}$ and $\delta_{d}$ are steps sizes, that are used to guarantee the algorithmic convergence.

\section{Iterative Algorithm}

In this section, we present the overall algorithm which is proposed to solve the original optimization problem (23). The algorithm compensates for the approximations made earlier while convexifying the optimization problem. Because the proposed solution of the reformulated problem iteratively updates the dual variables, the estimated DC bias vector is also updated at each iteration, so as to reflect the newest update of the values of the dual variables. The steps of the proposed algorithm are summarized in Algorithm 1 description.

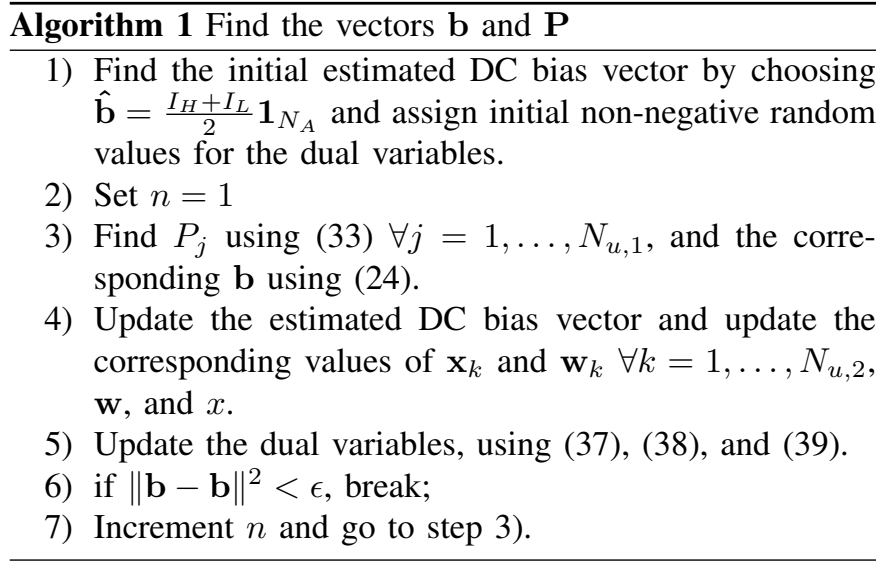

Remark 1. The main idea of Algorithm 1 is to update the dual variables along with the estimated DC bias vector in each iteration by equating it with the resulted DC bias vector from the previous iteration. This process continues until convergence. It is important to note that there is no unique values for the dual variables that can reach the optimal power. Such conclusion is due to the fact that the dual variables must be selected to achieve the corresponding constraints. Hence, in step 5) in Algorithm 1, we can find the $\lambda^{\prime}$ s using (37) by replacing the inequality with equality, which helps achieving the corresponding constraints.

\section{E. Baseline Algorithm}

For benchmarking purposes, we now propose a simple, yet feasible, solution to problem (23). In this approach, for simplicity, the DC bias values are assumed to be equal across all APs, i.e., $b_{i}=b$. Based on this assumption and within the bounds of the DC bias values, we find the maximum and minimum DC bias values that achieve the constraints in (23). It can be noticed that the minimum feasible DC bias value is the one that maximizes the sum rate, while the maximum feasible DC bias value is the one that maximizes the 
total harvested energy. Therefore, the idea of this approach is that, instead of weighting the utility functions, we weight the corresponding DC bias values. In other words, we linearly combine the minimum and the maximum DC bias vectors based on the given $\alpha$ value. After obtaining the fixed DC bias vector, we formulate a linear optimization problem to find the corresponding messages' power vector. If we scrutinize the constraints in (23), we see that the value of the DC bias $b$ must be increased if at least one of the constraints in $(23 \mathrm{c})$ is violated, while it must be decreased if at least one of the constraints in (23b) is violated. That means the constraints in (23c) and the constraint $b \geq \frac{I_{H}+I_{L}}{2}$ specify the minimum DC bias vector that achieves all the constraints. On the other hand, the constraints in (23b) and the constraint $b \leq I_{H}$ specify the maximum DC bias vector that achieves all the constraints. If the value reached while searching for the maximum DC bias value is found to be less than the value reached while searching for the minimum DC bias value, the problem of finding equal DC bias at all APs is then unfeasible.

To determine the minimum DC bias vector, we solve all the equations in (23c) under the assumption that all the values in the vector $\mathbf{b}$ are equal. For the $k$ th user, we find a solution for $b_{k}$ from the following equation

$$
\begin{array}{r}
b_{k} f \rho_{o e} \rho_{s e} V_{t} \mathbf{h}_{k}^{T} \mathbf{1}_{N_{A}} \ln \left(1+b_{k} \frac{\rho_{o e} \rho_{s e} \mathbf{h}_{k}^{T} \mathbf{1}_{N_{A}}}{I_{0}}\right) \geq E_{t h, k} \\
k=1, \ldots, N_{u, 2} .
\end{array}
$$

Equations (40) can be solved using any numerical methods such as Newton method. Define $\overline{\mathbf{b}} \in \mathbb{R}^{N_{u, 2} \times 1}$ as the vector that hosts the solutions of equations (40), the minimum DC bias vector can be given by

$$
\mathbf{b}_{\text {min }}=\max \left[\frac{I_{H}+I_{L}}{2}, \max (\overline{\mathbf{b}})\right] \mathbf{1}_{N_{A}} .
$$

To determine the maximum DC bias vector, we solve all the equations in (24) when $P_{j}=P_{j, \min }, j=1, \ldots, N_{u, 1}$. Therefore, the maximum DC bias vector is given by

$$
\mathbf{b}_{\max }=\min \left[I_{H}, I_{H}-\sqrt{\max (\overline{\mathbf{G}} \mathbf{P})}\right] \mathbf{1}_{N_{A}} .
$$

Based on a predefined $\alpha$, the DC bias solution of the baseline approach is given by

$$
\mathbf{b}=\alpha \mathbf{b}_{\min }+(1-\alpha) \mathbf{b}_{\max } .
$$

It can be seen that all the values in the solution vector $\mathbf{b}$ are equal. Because there is more than one solution of the messages' power vector $\mathbf{P}$ for the given DC bias vector, we formulate the following simple optimization problem to find an efficient power allocation

$$
\begin{aligned}
\max _{\mathbf{P}} & \sum_{j=1}^{N_{u, 1}} \gamma P_{j} \\
\text { s.t. } & P_{j} \geq P_{j, \min }, \quad j=1, \ldots, N_{u, 1} \\
& \overline{\mathbf{G}} \mathbf{P} \leq\left(I_{H} \mathbf{1}_{N_{A}}-\mathbf{b}\right)^{2}, i=1, \ldots, N_{A} \\
& \mathbf{P} \geq \mathbf{0} .
\end{aligned}
$$

Note that the vector $b$ in the constraints (44c) is given by (43). Problem (44) is a linear programming (LP) and can be solved easily by the CVX solver [47]. All the baseline approach procedures are summarized in Algorithm 2.

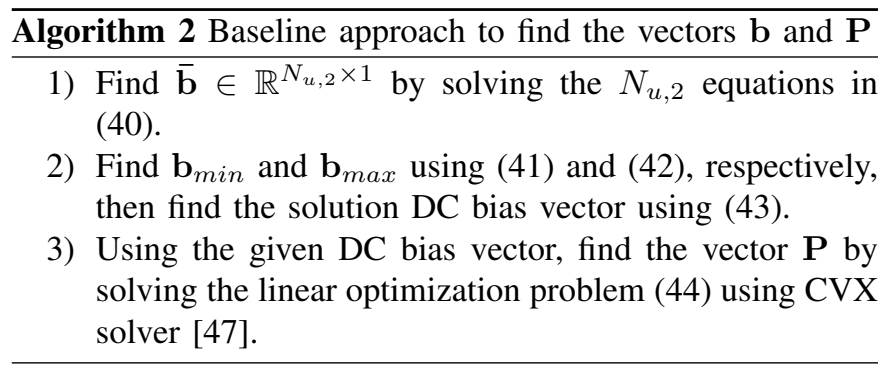

\section{F. Special cases}

In this section, we consider the two special cases of the weighted-sum formulated problem. In these cases, we focus on solving the problem that considers maximizing one of the two extreme utilities (i.e., either the total harvested energy or the sum rate) under the same considered constraints.

1) Maximizing the total harvested energy $(\alpha=0)$ : To maximize the harvested energy instead of the weighted sum function, we set $\alpha$ to 0 for both Algorithm 1 and the baseline approach. For the proposed Algorithm 1, the problem is interestingly cast and approximated as the following linear optimization problem

$$
\begin{array}{cl}
\max _{\mathbf{P}} & \left(x-\mathbf{w}^{T} \mathbf{P}\right) \\
\text { s.t. } & P_{j} \geq P_{j, \text { min }}, \quad j=1, \ldots, N_{u, 1} \\
& \mathbf{w}_{k}^{T} \mathbf{P} \leq m_{k} \quad k=1, \ldots, N_{u, 2} \\
& \overline{\mathbf{g}}_{i}^{T} \mathbf{P} \geq 0, \quad i=1, \ldots, N_{A} \\
& \overline{\mathbf{g}}_{i}^{T} \mathbf{P} \leq\left(\frac{I_{H}-I_{L}}{2}\right)^{2}, i=1, \ldots, N_{A} .
\end{array}
$$

Problem (45) can be solved efficiently using the CVX solver [47], without the need for the use of the dual decomposition method and the subgradient method. The steps of solving problem (45) are given in Algorithm 3.

For the baseline approach, the underlying algorithm (equal DC bias allocation) for solving problem (45) is given by

$$
\mathbf{b}=\min \left[I_{H}, I_{H}-\sqrt{\max \left(\overline{\mathbf{G}} \mathbf{P}_{\min }\right)}\right] \mathbf{1}_{N_{A}},
$$

which is the maximum feasible DC bias that achieves the constraints while maximizing the total harvested energy. The messages' power herein are given by $\mathbf{P}=\mathbf{P}_{\min }$.

2) Maximizing the Sum Rate $(\alpha=1)$ : The problem of sum rate maximization under the established constraints can be obtained by setting $\alpha=1$. The problem can be approximated as (32) with setting $\alpha=1$, and Algorithm (1) can be used to find the joint DC bias and power vector that maximize the sum rate function. Similarly, the power vector in the baseline approach for the sum rate maximization can be obtained by solving (44), where the DC bias vector is given by

$$
\mathbf{b}=\max \left[\frac{I_{H}+I_{L}}{2}, \max (\overline{\mathbf{b}})\right] \mathbf{1}_{N_{A}},
$$




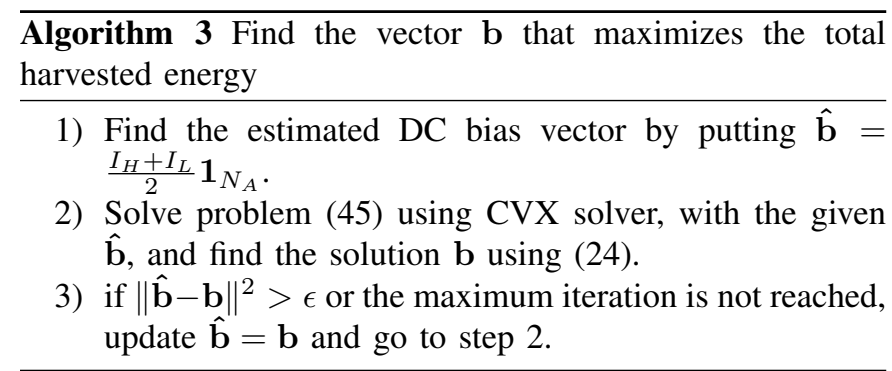

TABLE I

Simulation PARAMETERS

\begin{tabular}{lc}
\hline Parameter Name & Parameter Value \\
\hline & \\
VLC AP maximum bandwidth, $W$ & $20 \mathrm{MHz}$ \\
Physical area of a PD for IUs, $A_{p}(I U)$ & $1 \mathrm{~cm}^{2}$ \\
Solar cell physical area for EHUs, $A_{p}(E H U)$ & $0.04 \mathrm{~m}^{2}$ \\
Gain of optical filter, $g_{o} f$ & 1 \\
Half-intensity radiation angle, $\theta_{1 / 2}$ & $60^{\circ}$ \\
FoV semi-angle of PD, $\Theta$ & $40^{\circ}-65^{\circ}$ \\
Optical-to-electrical conversion factor of IUs, $\rho_{o e}$ & $0.53 \mathrm{~A} / \mathrm{W}$ \\
Optical-to-electrical conversion factor of EHUs, $\rho_{o e}$ & $0.4 \mathrm{~A} / \mathrm{W}$ \\
Refractive index, $n$ & 1.5 \\
Maximum input bias current, $I_{H}$ & $12 \mathrm{~mA}$ \\
Minimum input bias current, $I_{L}$ & $0 \mathrm{~A}$ \\
Fill factor, $f$ & 0.75 \\
Electric-to-optical conversion factor, $\rho_{s e}$ & $10 \mathrm{~W} / \mathrm{A}$ \\
Thermal voltage, $V_{t}$ & $25 \mathrm{mV}$ \\
Dark saturation current of the PD, $I_{0}$ & $0.1 \mathrm{nA}$ \\
Noise power spectral density, $N_{0}$ & $10^{-22} \mathrm{~A} / \mathrm{Hz}$ \\
Room size & $8 \times 8 \mathrm{~m}$ \\
Room height & $3 \mathrm{~m}$ \\
User height & $0.85 \mathrm{~m}$ \\
Number of APs & $4 \times 4$ \\
Minimum IUs data rate, $R_{t h, j}, j=1, \ldots, N_{u, 1}$ & $10(\mathrm{Mbits} / \mathrm{sec})$ \\
Minimum EHUs energy, $E_{t h, k}, k=1, \ldots, N_{u, 2}$ & $1 \mu \mathrm{Joule}$ \\
\hline
\end{tabular}

which is the minimum equal DC bias that achieves the constraints while maximizing the sum rate.

\section{G. Computational Complexity}

This section discusses the computational complexity of both the Algorithm 1 and the baseline approach. It is shown in [45], [48] that the complexity of the subgradient approach is a polynomial function of the number of the dual variables, which is $M=N_{u, 1}+N_{u, 2}+N_{A}$. Besides, in each iteration, we need to update the estimated DC bias and the corresponding variables $\mathbf{x}_{k}, k=1, \ldots, N_{u, 2}$ and $\mathbf{w}_{k}, k=1, \ldots, N_{u, 2}$. This means that for updating the DC bias vector, the number of the updated variables in each iteration is $N_{A} \times N_{u, 2}+N_{A} \times N_{u, 1}$. Therefore, Algorithm 1 has a computational complexity in the order of $O\left(I_{R}\left(M+N_{A} \times N_{u, 2}+N_{A} \times N_{u, 1}\right)\right)$, where $I_{R}$ is the iterations' number needed for Algorithm 1 convergence.

On the other hand, the computational complexity of the proposed baseline approach is mainly due to solving a LP optimization problem, which is shown to be bounded by $O\left(n^{2} l\right)$, where $l$ is the number of constraints and $n=N_{u, 1}$ is the number of variables [46].

\section{Simulations Results}

This section evaluates the performance of the proposed algorithms by illustrating how the weight $\alpha$, the number of

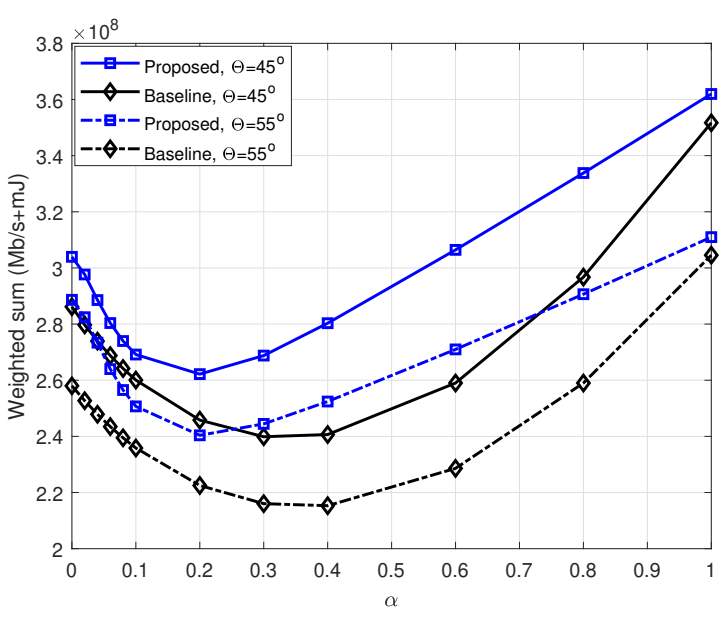

Fig. 3. Comparison between the proposed algorithm and the proposed baseline by plotting the weighted sum function versus the weight $\alpha$ for different users' FoV, $N_{u, 1}=5, N_{u, 2}=5$, and $\omega=\frac{10^{-3}}{12 \times W}$.

users (either IUs or EHUs), and the FoV affect the total harvested energy, sum rate, and the weighted sum function. All the simulation results are implemented under the simulation parameters given in Table I, similar to [30], [35], and [39]. Consider an $8 \times 8 \times 3 \mathrm{~m}^{3}$ room equipped with 16 VLC APs that are at ceiling level, and serve several IUs and EHUs. Monte-Carlo simulations are used to assess the performance of the proposed algorithms, where every point in the numerical results is the average of implementing 100 different user realizations.

Fig. 3 compares the proposed Algorithm 1 with the proposed baseline approach by plotting the weighted sum function versus $\alpha$. The figure shows that the proposed Algorithm 1 outperforms the proposed baseline approach for all different weights and different users' FoV. The figure further shows that the weighted sum function is maximized when $\alpha=0$ or 1 , i.e., when the weighted sum function is just the total harvested energy or the sum rate function, respectively. Such performance behavior can be justified by the fact that when $\alpha$ is small (i.e. when $\alpha \leq 0.3$ ), the dominating function is the total harvested energy, and hence the increase in $\alpha$ decreases the weighted sum, while when $\alpha$ is large $(\alpha \geq 0.4)$ the dominating function is the sum rate and, hence, the increase in $\alpha$ increases the weighted sum function.

To show how the weight $\alpha$ affects the sum rate and the total harvested energy, we plot the sum rate function versus $\alpha$ in Fig. 4, and the total harvested energy versus $\alpha$ in Fig. 5. It can be seen from both figures that as the weight increases, the sum rate increases and the total harvested energy decreases, but with a decreased rate in the proposed approach. The figures also show that for the different values of $\alpha$, as the sum rate increases (as shown in Fig. 4), the total harvested energy decreases (as shown in Fig. 5). These results confirm that the sum rate and the total harvested energy functions exhibit an opposite behavior, and can be controlled by allocating the DC bias, since decreasing the DC bias decreases the total harvested energy and preserves much power for transmitting 


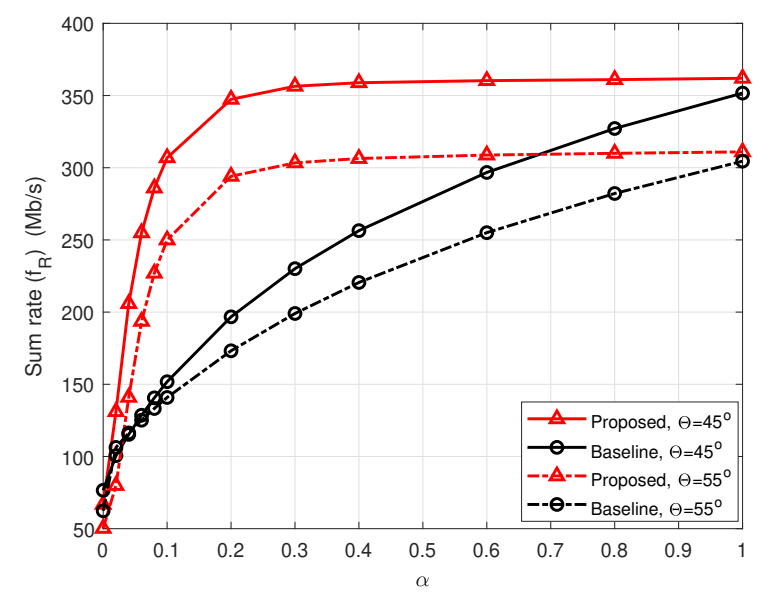

Fig. 4. The sum rate function versus $\alpha$ for different users' FoV.

data. Both figures further show that at some values of $\alpha$, if the proposed baseline approach outperforms the proposed Algorithm 1 at one utility function (either the sum rate or the total harvested energy), it provides much less performance at the same points at the other utility function. Both figures also show that in the proposed approach, the impact of $\alpha$ on sum rate and harvested energy functions decreases as $\alpha$ increases from 0 to 1 , and this is why we measure more points when $\alpha$ is less than 0.1 . We observed that the numerical choice of $\omega$ affects the decrease (and increase) rate of the sum rate and the harvested energy. To decrease this rate of increasing (or decreasing), the value of $\omega$ should be adapted with $\alpha$. However, this is not our interest, since we can confine our study in the range of $\alpha$ effectiveness.

Figs. 3, 4, and 5 show that the performance of the utility functions is better at lower values of FOVs, i.e., the $45^{\circ}$ FOV case as compared to the $55^{\circ}$ FOV case. Such result is

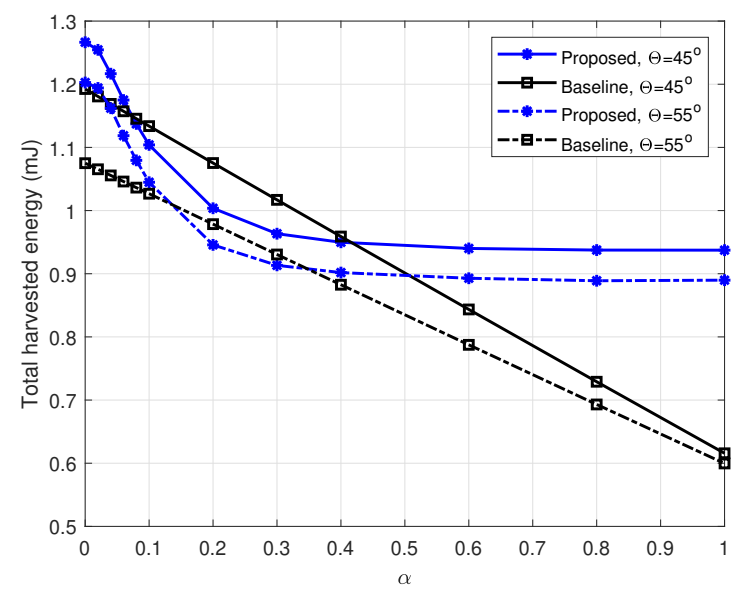

Fig. 5. The total harvested energy function versus $\alpha$ for different users' FoV.

further illustrated in Fig. 6 with different number of EHUs and IUs. Equation (4) further justifies this fact, since if the FoV $(\Theta)$ increases between $0^{\circ}$ and $90^{\circ}$, the channel quality (the channel value) decreases significantly. On the other hand,

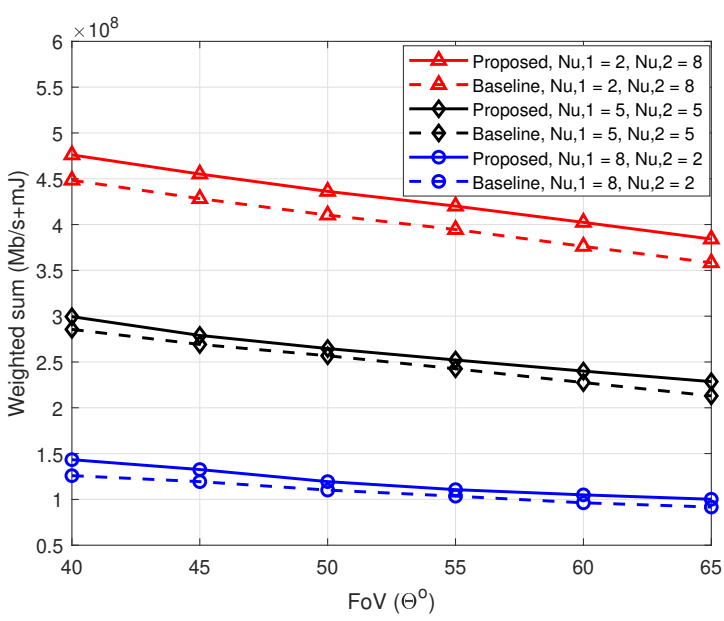

Fig. 6. The weighted sum function versus users' FoV with different number of EHUs and IUs $\alpha=0.1$ and $\omega=\frac{10^{-3}}{12 \times W}$.

from equation in (4), decreasing the user's FoV decreases the probability of coverage at that user. As a result, we can conclude that if the users' FoV is adjustable, decreasing its value subject to having at least one VLC AP in the FoV of that user would indeed increase the network harvested energy. The figures also show that the proposed iterative Algorithm 1 outperforms the proposed baseline approach with all the different users' FoV and different number of IUs and EHUs. To balance between the sum rate and the total harvested energy functions, we select $\alpha=0.1$, where as shown in Figs. 4 and $5 \alpha=0.1$ provides a sensible trade-off between the sum rate and the total harvested energy functions. Fig. 6 further shows the behavior of the weighted sum function when the number of EHUs is dominating, when the number of both EHUs and IUs are equal, and when the number of IUs is dominating. It can be seen that the effect of increasing number of EHUs is higher than the effect of increasing the number of IUs. Increasing the number of EHUs would increase the total harvested energy linearly, while increasing the number of IUs would increase the sum rate but at a decreasing rate. This is because the available bandwidth and power per information user depends on the number of users, while the harvested energy is not a function of the bandwidth and the $\mathrm{AC}$ power. Another reason is because of the value of $\omega$, which should be ideally adapted with changing the number of users.

Fig. 7 studies the effect of the users' FoV and the number of users on the sum rate function. In this figure, we optimize the sum rate under QoS constraints which can be implemented by setting $\alpha=1$ in the weighted sum function. As expected, decreasing the users' FoV, increasing the IUs, or decreasing the number of EHUs improve the sum rate as shown in Fig. 7. The figure also shows that the proposed Algorithm 1 outperforms the proposed baseline approach or the equal DC bias allocation approach at the different scenarios considered in the figure, especially when the number of IUs is high.

Fig. 8 studies the effect of the users' FoV and the number of users on the total harvested energy function. In this figure, we use Algorithm 3 instead of Algorithm 1 to solve the 


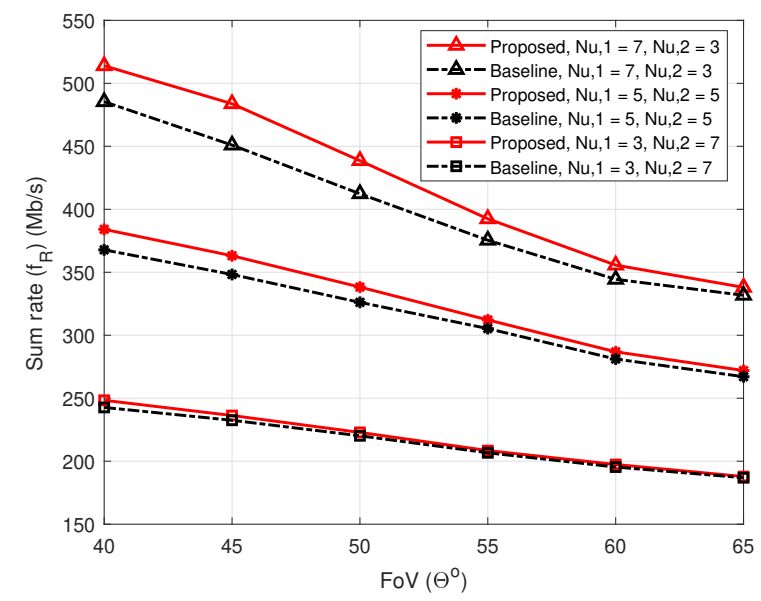

Fig. 7. The sum rate versus users' FoV with different number of EHUs and IUs, $\alpha=1$.

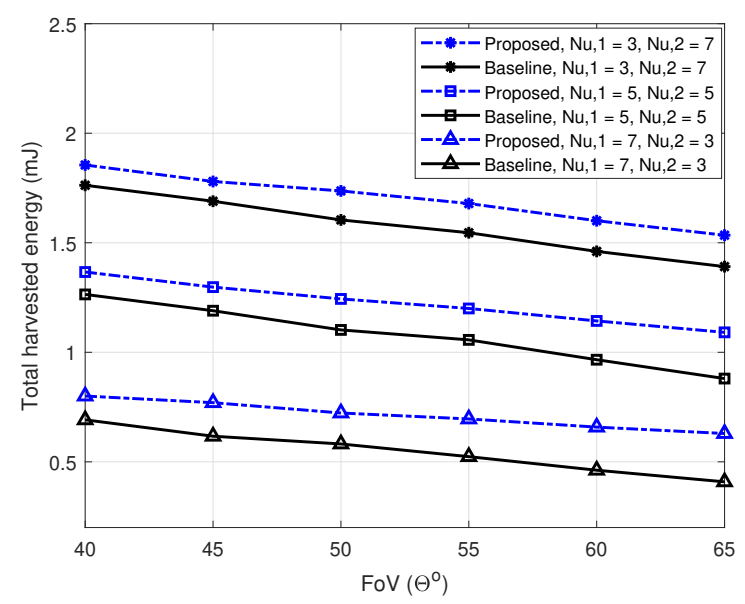

Fig. 8. The total harvested energy versus users' FoV with different number of EHUs and IUs, $\alpha=0$.

optimization problem, which is a special case that can be implemented when $\alpha=0$ in the weighted sum function. As expected, the figure shows that decreasing the users' FoV, increasing the IUs, or decreasing the number of EHUs lead to increasing the total harvested energy. The figure also shows that the proposed Algorithm 3 outperforms the proposed baseline approach (i.e., the equal DC bias allocation approach) at the different scenarios considered in the figure.

To illustrate the convergence of the iterative algorithm proposed to compensate for the used approximations, Fig. 9 studies the behavior of Algorithm 3 and plots the total harvested energy at all EHUs versus the number of iterations, for two values of the FoV and different numbers of IUs and EHUs. The figure shows the fast convergence of Algorithm 3 for all values of FoV for the different number of users, which further highlight the numerical efficiency of our proposed algorithm.

Fig. 10 plots the sum rate as a function of the percentage of number of EHUs out of the total number of users, also denoted by $\eta$ (i.e. $\eta=\frac{N_{u, 2}}{N_{u, 2}+N_{u, 1}}$ ). This figure shows that

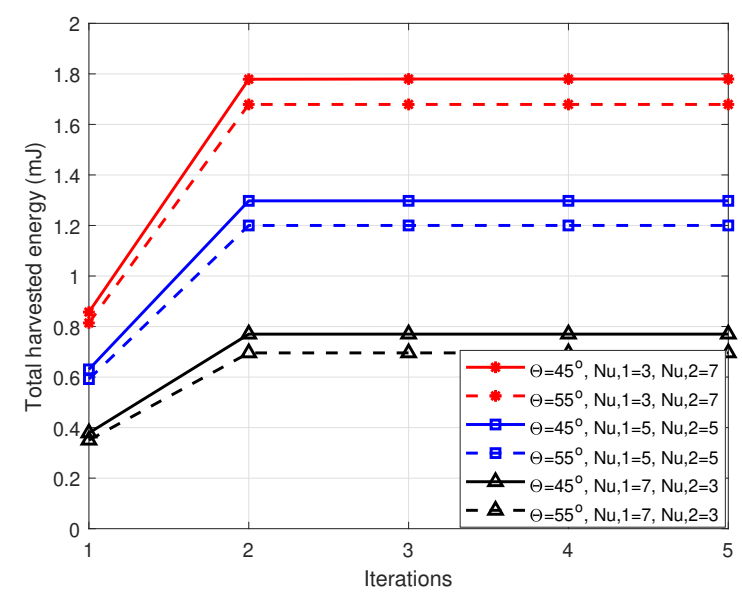

Fig. 9. The total harvested energy versus number of iterations with different users' FoV and different number of IUs and EHUs, $\alpha=0$.

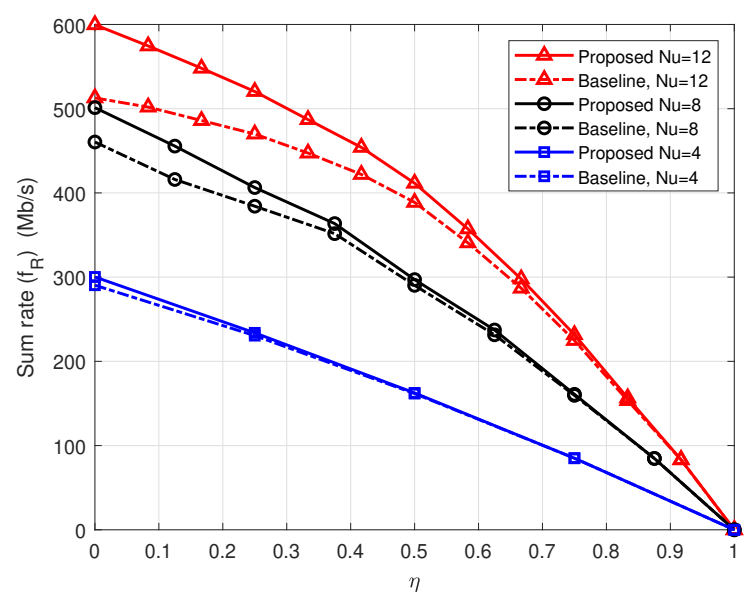

Fig. 10. The sum rate versus $\eta$ (the percentage of EHUs out of total number of users $N_{u}$ ), with different total number of users, FoV $=45^{\circ}$.

the sum rate decreases as $\eta$ increases, because increasing the EHUs or decreasing the IUs lead to decreasing the sum rate. This figure also shows that increasing the total number of IUs increases the sum rate but with a slower rate, since the rate achieved by increasing $N_{u}=4$ to $N_{u}=8$ is around double the rate achieved by increasing $N_{u}=8$ to $N_{u}=12$. This is because adding one user to the system decreases the assigned power (on average) for the existing users for a given fixed transmit power.

Lastly, Fig. 11 shows that as the fraction of EHUs increases, the total harvested energy increases. As expected, this is mainly due to two main reasons. Firstly, for a fixed number of users $N_{u}$, as the fraction of EHUs increases, the number of EHUs increases, which adds to the total harvested energy. Secondly, decreasing the number of IUs leads to decreasing the number of constraints in (23b), which increases the search space of (23b); thereby increasing the objective function. The figure further shows that, if $\eta=1$ (i.e. when all users are EHUs), all the APs operate with a highest DC bias (i.e. $\left.b_{i}=I_{H}, \quad i=1, \ldots, N_{A}\right)$, and so both the iterative algorithm 


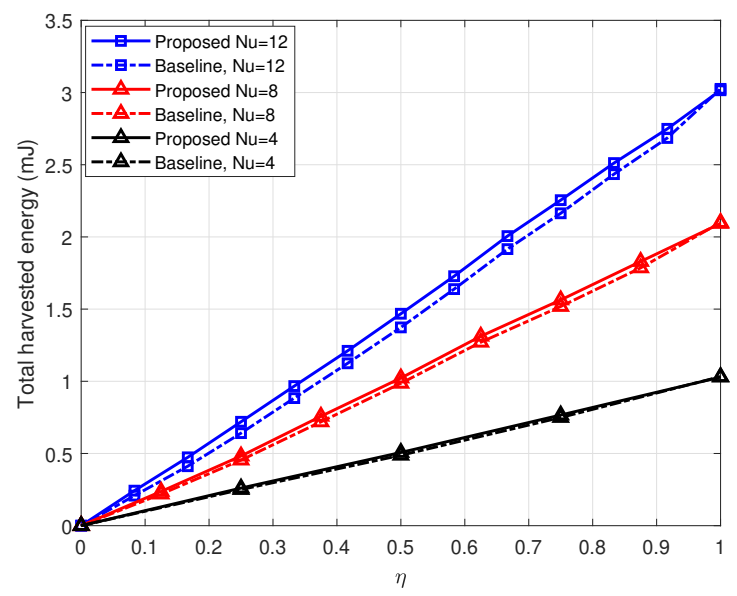

Fig. 11. The total harvested energy versus $\eta$ (the percentage of EHUs out of total number of users $N_{u}$ ) with different total number of users, FoV $=45^{\circ}$.

and the baseline achieve the same performance. On the other hand, if $\eta=0$ (i.e., when all users are IUs), the total harvested energy becomes zero.

\section{CONCLUSIONS}

VLC-based systems are expected to play a major role in achieving the ambitious metrics of next generation indoor wireless networks. This paper considers a VLC setup which considers the coexistence of both IUs and EHUs, and addresses the problem of maximizing a weighted sum of the total harvested energy and the sum rate by means of properly adjusting the DC bias values at the coordinating VLC APs and the messags' power vector subject to QoS constraints (minimum required data rate at IUs and minimum required harvested energy at EHUs). The paper solves such a difficult problem using an iterative algorithm by first using an inner convex approximation, and then by properly compensating for the approximation in an outer loop. Simulation results show that an appreciable, balanced performance improvement in both utility functions (the sum rate and the total harvested energy) can be achieved by jointly optimizing the DC bias vector and the messages' power vector.

\section{ACKNOWLEDGMENT}

This work was supported by the Deanship of Scientific Research in King Fahd University of Petroleum \& Minerals (KFUPM) through grant number SB181019. Hayssam Dahrouj would like to thank Effat University in Jeddah, Saudi Arabia, for supporting the research reported in this paper through the Research and Consultancy Institute.

\section{REFERENCES}

[1] M. Obeed, A. M. Salhab, S. A. Zummo, and M.-S. Alouini, "DCbias allocation in cooperative VLC networks via joint information and energy transfer," in Proc. IEEE Global Commun. Conf. (Globecom), Abu Dhabi, UAE, 2017.

[2] M. Obeed, A. M. Salhab, M.-S. Alouini, and S. A. Zummo, "On optimizing VLC networks for downlink multi-user transmission: A survey," IEEE Commun. Surveys Tuts, 2019.
[3] H. Elgala, R. Mesleh, and H. Haas, "Indoor optical wireless communication: potential and state-of-the-art," IEEE Commun. Mag., vol. 49, no. 9,2011

[4] P. H. Pathak, X. Feng, P. Hu, and P. Mohapatra, "Visible light communication, networking, and sensing: A survey, potential and challenges," IEEE Commun. Surveys Tuts, vol. 17, no. 4, pp. 2047-2077, 2015.

[5] I. Stefan, H. Burchardt, and H. Haas, "Area spectral efficiency performance comparison between VLC and RF femtocell networks," in 2013 IEEE International Conference on Communications (ICC). IEEE, jun 2013.

[6] J. Miyakoshi, "Cellular and molecular responses to radio-frequency electromagnetic fields," Proc. IEEE, vol. 101, no. 6, pp. 1494-1502, 2013.

[7] H. Haas, L. Yin, Y. Wang, and C. Chen, "What is Li-Fi?" J. Lightw. Technol., vol. 34, no. 6, pp. 1533-1544, 2016.

[8] S. Dimitrov and H. Haas, Principles of LED light communications: towards networked Li-Fi. Cambridge University Press, 2015.

[9] S. H. Kim, H. H. Park, Y. H. Song, H. J. Park, J. B. Kim, S. R. Jeon, H. Jeong, M. S. Jeong, and G. M. Yang, "An improvement of light extraction efficiency for gan-based light emitting diodes by selective etched nanorods in periodic microholes," Opt. Express, vol. 21, no. 6 , pp. 7125-7130, 2013.

[10] Y. Song and M. Larry Lee, "Room temperature electroluminescence from light-emitting diodes based on in 0 . 5ga0. 5as/gap self-assembled quantum dots," Appl. Phys. Lett., vol. 100, no. 25, p. 251904, 2012.

[11] K. Yung, H. Liem, H. Choy, and W. Lun, "Degradation mechanism beyond device self-heating in high power light-emitting diodes," J. Appl. Phys., vol. 109, no. 9, p. 094509, 2011.

[12] Z. Wang, D. Tsonev, S. Videv, and H. Haas, "On the design of a solarpanel receiver for optical wireless communications with simultaneous energy harvesting," IEEE J. Sel. Areas Commun., vol. 33, no. 8, pp. 1612-1623, 2015.

[13] R. King, D. Bhusari, D. Larrabee, X.-Q. Liu, E. Rehder, K. Edmondson, H. Cotal, R. Jones, J. Ermer, C. Fetzer et al., "Solar cell generations over 40\% efficiency," Prog. Photovoltaics Res. Appl., vol. 20, no. 6, pp. 801-815, 2012.

[14] M. Obeed, A. M. Salhab, S. A. Zummo, and M.-S. Alouini, "Joint optimization of power allocation and load balancing for hybrid VLC/RF networks," IEEE/OSA J. Opt. Commun. and Netw., vol. 10, no. 5, pp. 553-562, 2018

[15] Y. Wang, D. A. Basnayaka, and H. Haas, "Dynamic load balancing for hybrid Li-Fi and RF indoor networks," in Proc. IEEE Int. Conf. Commun. Workshops (ICCW), London, UK, 2015, pp. 1422-1427.

[16] M. Obeed, A. M. Salhab, S. A. Zummo, and M.-S. Alouini, "Joint load balancing and power allocation for hybrid VLC/RF networks," in Proc. IEEE Global Commun. Conf. (Globecom), Singapore, 2017.

[17] Y. Wang, X. Wu, and H. Haas, "Load balancing game with shadowing effect for indoor hybrid LiFi/RF networks," IEEE Trans. Wireless Commun., vol. 16, no. 4, pp. 2366-2378, 2017.

[18] X. Li, F. Jin, R. Zhang, J. Wang, Z. Xu, and L. Hanzo, "Users first: User-centric cluster formation for interference-mitigation in visible-light networks," IEEE Trans. Wireless Commun., vol. 15, no. 1, pp. 39-53, 2016.

[19] M. Obeed, A. M. Salhab, S. A. Zummo, and M.-S. Alouini, "New algorithms for energy-efficient VLC networks with user-centric cell formation," IEEE Trans. Green Commun. Netw., 2018.

[20] X. Li, R. Zhang, and L. Hanzo, "Cooperative load balancing in hybrid visible light communications and WiFi," IEEE Trans. Commun., vol. 63 , no. 4, pp. 1319-1329, 2015.

[21] C. Chen, D. Tsonev, and H. Haas, "Joint transmission in indoor visible light communication downlink cellular networks," in Globecom Workshops (GC Wkshps), 2013 IEEE. IEEE, 2013, pp. 1127-1132.

[22] T. V. Pham and A. T. Pham, "Max-min fairness and sum-rate maximization of MU-VLC local networks," in 2015 IEEE Globecom Workshops (GC Wkshps). IEEE, dec 2015

[23] L. P. Qian, Y. J. Zhang, and J. Huang, "Mapel: Achieving global optimality for a non-convex wireless power control problem," IEEE Trans. Wireless Commun., vol. 8, no. 3, pp. 1553-1563, 2009.

[24] H. Dahrouj, W. Yu, and T. Tang, "Power spectrum optimization for interference mitigation via iterative function evaluation," EURASIP $J$. Wireless Commun. Netw., vol. 2012, no. 1, p. 244, 2012.

[25] R. Jiang, Q. Wang, H. Haas, and Z. Wang, "Joint user association and power allocation for cell-free visible light communication networks," IEEE J. Sel. Areas Commun., 2017.

[26] H. Shen, Y. Deng, W. Xu, and C. Zhao, "Rate-maximized zero-forcing beamforming for vlc multiuser miso downlinks," IEEE Photon. J., vol. 8 , no. 1 , pp. $1-13,2016$. 
[27] X. Wu, M. Safari, and H. Haas, "Joint optimisation of load balancing and handover for hybrid LiFi and WiFi networks," in Proc. IEEE Wireless Commun. and Netw. Conf. (WCNC). San Francisco, USA: IEEE, 2017, pp. 1-5.

[28] Y. Wang, X. Wu, and H. Haas, "Load balancing game with shadowing effect for indoor hybrid LiFi/RF networks," IEEE Trans. Wireless Commun., vol. 16, no. 4, pp. 2366-2378, Apr. 2017.

[29] C. Li, W. Jia, Q. Tao, and M. Sun, "Solar cell phone charger performance in indoor environment," in Bioengineering Conference (NEBEC), 2011 IEEE 37th Annual Northeast. IEEE, 2011, pp. 1-2.

[30] T. Rakia, H.-C. Yang, F. Gebali, and M.-S. Alouini, "Optimal design of dual-hop VLC/RF communication system with energy harvesting," IEEE Commun. Lett., vol. 20, no. 10, pp. 1979-1982, 2016.

[31] — "Dual-hop VLC/RF transmission system with energy harvesting relay under delay constraint," in Globecom Workshops (GC Wkshps), 2016 IEEE. IEEE, 2016, pp. 1-6.

[32] Y. Li, N. Huang, J. Wang, Z. Yang, and W. Xu, "Sum rate maximization for VLC systems with simultaneous wireless information and power transfer," IEEE Photon. Technol. Lett., vol. 29, no. 6, pp. 531-534, 2017.

[33] A. M. Abdelhady, O. Amin, A. Chaaban, and M. S. Alouini, "Resource allocation for outdoor visible light communications with energy harvesting capabilities," in 2017 IEEE Globecom Workshops (GC Wkshps), Dec 2017, pp. 1-6.

[34] G. Pan, H. Lei, Z. Ding, and Q. Ni, "On 3-D hybrid VLC-RF systems with light energy harvesting and OMA scheme over RF links," 2017.

[35] P. D. Diamantoulakis, G. K. Karagiannidis, and Z. Ding, "Simultaneous lightwave information and power transfer (SLIPT)," IEEE Trans. Green Commun. Netw., 2018.

[36] J. Fakidis, S. Videv, S. Kucera, H. Claussen, and H. Haas, "Indoor optical wireless power transfer to small cells at nighttime," J. Lightw. Technol., vol. 34, no. 13, pp. 3236-3258, 2016.

[37] J. Fakidis, S. Videv, H. Helmers, and H. Haas, "0.5-gb/s OFDM-based laser data and power transfer using a GaAs photovoltaic cell," IEEE Photon. Technol. Lett., vol. 30, no. 9, pp. 841-844, may 2018.

[38] G. Pan, P. D. Diamantoulakis, Z. Ma, Z. Ding, and G. K. Karagiannidis, "Simultaneous lightwave information and power transfer: Policies, techniques, and future directions," IEEE Access, vol. 7, pp. 28250 28257, 2019.

[39] R. Zhang, H. Claussen, H. Haas, and L. Hanzo, "Energy efficient visible light communications relying on amorphous cells," IEEE J. Sel. Areas Commun., vol. 34, no. 4, pp. 894-906, 2016.

[40] M. Obeed, A. M. Salhab, S. A. Zummo, and M.-S. Alouini, "Joint power allocation and cell formation for energy-efficient VLC networks," in IEEE International Conf. on Commun. (ICC), Kansas, USA, 2018.

[41] J. M. Kahn and J. R. Barry, "Wireless infrared communications," Proc. IEEE, vol. 85, no. 2, pp. 265-298, 1997.

[42] T. Komine and M. Nakagawa, "Fundamental analysis for visiblelight communication system using LED lights," IEEE Trans. Consum. Electron., vol. 50, no. 1, pp. 100-107, 2004.

[43] J.-B. Wang, Q.-S. Hu, J. Wang, M. Chen, and J.-Y. Wang, "Tight bounds on channel capacity for dimmable visible light communications," $J$. Lightw. Technol., vol. 31, no. 23, pp. 3771-3779, 2013.

[44] X. Li, Y. Huo, R. Zhang, and L. Hanzo, "User-centric visible light communications for energy-efficient scalable video streaming," IEEE Trans. Green Commun. Netw., vol. 1, no. 1, pp. 59-73, 2017.

[45] M. Kashef, M. Ismail, M. Abdallah, K. A. Qaraqe, and E. Serpedin, "Energy efficient resource allocation for mixed RF/VLC heterogeneous wireless networks," IEEE J. Sel. Areas Commun., vol. 34, no. 4, pp. 883-893, 2016.

[46] S. Boyd and L. Vandenberghe, Convex Optimization. Cambridge University Press, 2004.

[47] M. Grant and S. Boyd, "CVX: Matlab software for disciplined convex programming," 2008

[48] M. S. Alam, J. W. Mark, and X. S. Shen, "Relay selection and resource allocation for multi-user cooperative OFDMA networks," IEEE Trans. Wireless Commun., vol. 12, no. 5, pp. 2193-2205, 2013.

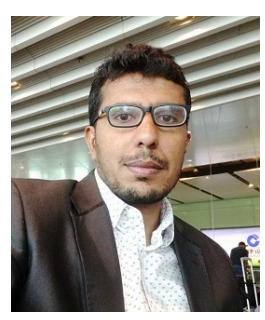

Mohanad Obeed (S'17) received the B.Eng. degree in computer and communication engineering from Taiz University, Taiz, Yemen, in 2008, the M.Sc. and the $\mathrm{Ph} . \mathrm{D}$. degree in electrical engineering from King Fahd University of Petroleum and Minerals (KFUPM), Dhahran, Saudi Arabia, in 2016 and 2019, respectively. From July 2017 to July 2019, he was a visiting student at King Abdullah University of Science and Technology (KAUST) under the supervision of Mohamed-Slim Alouini. His research interests include visible light communications, cooperative communication, resource allocation, convex optimization, physical layer security, and energy harvesting.

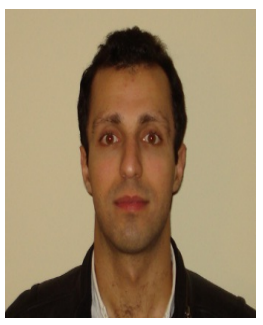

Hayssam Dahrouj (S'02, M'11, SM'15) received his B.E. degree (with high distinction) in computer and communications engineering from the American University of Beirut (AUB), Lebanon, in 2005 , and his Ph.D. degree in electrical and computer engineering from the University of Toronto (UofT), Canada, in 2010. In May 2015, he joined the Department of Electrical and Computer Engineering at Effat University as an assistant professor, and also became a visiting scholar at King Abdullah University of Science and Technology (KAUST). Between April 2014 and May 2015, he was with the Computer, Electrical and Mathematical Sciences and Engineering group at KAUST as a research associate. Prior to joining KAUST, he was an industrial postdoctoral fellow at UofT, in collaboration with BLiNQ Networks Inc., Kanata, Canada, where he worked on developing practical solutions for the design of non-line-of sight wireless backhaul networks. His contributions to the field led to five patents. During his doctoral studies at UofT, he pioneered the idea of coordinated beamforming as a means of minimizing intercell interference across multiple base-stations. The journal paper on this subject was ranked second in the 2013 IEEE Marconi paper awards in wireless communications. Prof. Dahrouj is the recipient both of the faculty award of excellence in research, and of the faculty award of excellence in teaching (at the university level) in May 2017. $\mathrm{He}$ is a senior member of the IEEE. His main research interests include multi-base signal processing in cloud-radio access networks, visible light communications, free-space optics, machine learning, convex optimization, and distributed algorithms.

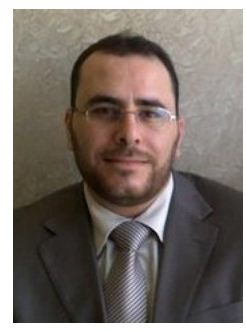

Anas M. Salhab (S'11-M'14-SM'17) received the B.Sc. degree in electrical engineering from Palestine Polytechnic University, Hebron, Palestine, in 2004, the M.Sc. degree in electrical engineering from Jordan University of Science and Technology, Irbid, Jordan, in 2007, and the Ph.D. degree from King Fahd University of Petroleum and Minerals (KFUPM), Dhahran, Saudi Arabia, in 2013. From 2013 to 2014, he was a Postdoctoral Fellow with the Electrical Engineering Department, KFUPM. He is currently an Assistant Professor and the Director of the Sponsored Research Office with the Deanship of Scientific Research, KFUPM. His research interest spans special topics in modeling and performance analysis of wireless communication systems, including cooperative relay networks, cognitive radio relay networks, free space optical networks, visible light communications, and co-channel interference. He was selected as an Exemplary Reviewer by the IEEE WIRELESS COMMUNICATIONS LETTERS for his reviewing service in 2014. Recently, he has been nominated by ISI and SCOPUS among the top 10 faculty and researchers in KFUPM (2013-2018) for the "Excellence Award for Scientific Production (Engineering and Technology Field)" offered by the Saudi Digital Library, Ministry of Education, Saudi Arabia. 


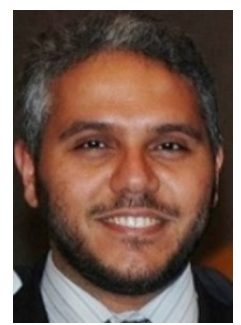

Salam A. Zummo (M'00-SM'08) received the B.Sc. and M.Sc. degrees in electrical engineering from the King Fahd University of Petroleum and Minerals (KFUPM), Dhahran, Saudi Arabia, in 1998 and 1999, respectively, and the Ph.D. degree from the University of Michigan, Ann Arbor, USA, in 2003. He is currently a Professor with the Electrical Engineering Department, KFUPM. Prof. Zummo has six issued U.S. patents and authored over 100 papers in reputable journals and conference proceedings. His research interests are in the area of wireless communications, including cooperative diversity, cognitive radio, multiuser diversity, scheduling, MIMO systems, error control coding, multihop networks, and interference modeling and analysis in wireless systems. He received the Saudi Ambassador Award for early Ph.D. completion in 2003, and the British Council/BAE Research Fellowship Awards in 2004 and 2006. He also received the KFUPM Excellence in Research Award from 2011 to 2012 .

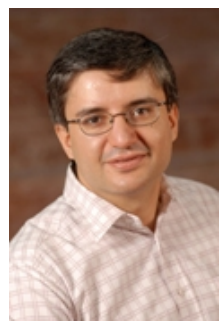

Mohamed-Slim Alouini (S'94, M'98, SM'03, F'09) was born in Tunis, Tunisia. He received the $\mathrm{Ph} . \mathrm{D}$. degree in Electrical Engineering from the California Institute of Technology (Caltech), Pasadena, CA, USA, in 1998. He served as a faculty member in the University of Minnesota, Minneapolis, MN, USA, then in the Texas A\&M University at Qatar, Education City, Doha, Qatar before joining King Abdullah University of Science and Technology

(KAUST), Thuwal, Makkah Province, Saudi Arabia as a Professor of Electrical Engineering in 2009. His current research interests include the modeling, design, and performance analysis of wireless communication systems. 